\title{
Relational Formal Characterization of Rough Sets
}

\author{
Adam Grabowski \\ Institute of Informatics \\ University of Białystok \\ Akademicka 2, 15-267 Białystok, Poland
}

\begin{abstract}
Summary. The notion of a rough set, developed by Pawlak [10, is an important tool to describe situation of incomplete or partially unknown information. In this article, which is essentially the continuation of [6], we try to give the characterization of approximation operators in terms of ordinary properties of underlying relations (some of them, as serial and mediate relations, were not available in the Mizar Mathematical Library). Here we drop the classical equivalence- and tolerance-based models of rough sets 12 trying to formalize some parts of [19] following also [18] in some sense (Propositions 1-8, Corr. 1 and 2 ; the complete description is available in the Mizar script). Our main problem was that informally, there is a direct correspondence between relations and underlying properties, in our approach however [7, which uses relational structures rather than relations, we had to switch between classical (based on pure set theory) and abstract (using the notion of a structure) parts of the Mizar Mathematical Library. Our next step will be translation of these properties into the pure language of Mizar attributes.
\end{abstract}

MML identifier: ROUGHS_2, version: 8.1.01 5.8.1171

The notation and terminology used in this paper have been introduced in the following articles: [13], [11, [5], [1], [2], [14, [3], 99, [16], 6], [15], [17, [8], and [4].

\section{Preliminaries}

One can verify that there exists a relational structure which is non empty and void.

Now we state the propositions:

(C) 2013 University of Białystok CC-BY-SA License ver. 3.0 or later ISSN 1426-2630(Print), 1898-9934(Online) 
(1) Let us consider a total non empty relational structure $R$ and an element $x$ of $R$. Then $x \in$ field the internal relation of $R$.

(2) Let us consider a non empty 1-sorted structure $R$ and a subset $X$ of $R$. Then $\{x$ where $x$ is an element of $R: \emptyset \subseteq X\}=\Omega_{R}$. Proof: $y \in$ $\{x$ where $x$ is an element of $R: \emptyset \subseteq X\}$.

(3) Let us consider a 1-sorted structure $R$ and a subset $X$ of $R$. Then $\{x$ where $x$ is an element of $R: \emptyset$ meets $X\}=\emptyset_{R}$.

\section{Missing Ordinary Properties of Binary Relations}

Let $R$ be a binary relation and $X$ be a set. We say that $R$ is serial in $X$ if and only if

(Def. 1) Let us consider an element $x$. Suppose $x \in X$. Then there exists an element $y$ such that

(i) $y \in X$, and

(ii) $\langle x, y\rangle \in R$.

We say that $R$ is serial if and only if

(Def. 2) $\quad R$ is serial in field $R$.

Let $R$ be a relational structure. We say that $R$ is serial if and only if

(Def. 3) the internal relation of $R$ is serial in the carrier of $R$.

One can check that every relational structure which is reflexive is also serial.

Let $R$ be a non empty relational structure. One can verify that $R$ is serial if and only if the condition (Def. 4) is satisfied.

(Def. 4) Let us consider an element $x$ of $R$. Then there exists an element $y$ of $R$ such that $x \leqslant y$.

Let us observe that every relational structure which is total is also serial and every relational structure which is serial is also total.

Let $R$ be a non empty serial relational structure and $x$ be an element of $R$. Let us note that $[x]_{\text {the internal relation of } R}$ is non empty.

Now we state the proposition:

(4) Let us consider a non empty reflexive relational structure $R$ and an element $x$ of $R$. Then $x \in[x]_{\alpha}$, where $\alpha$ is the internal relation of $R$. The theorem is a consequence of (1).

Let $R$ be a non empty reflexive relational structure and $x$ be an element of

$R$. Note that $[x]_{\text {the internal relation of } R}$ is non empty.

Let $R$ be a binary relation and $X$ be a set. We say that $R$ is mediate in $X$ if and only if

(Def. 5) Let us consider elements $x, y$. Suppose $x, y \in X$. If $\langle x, y\rangle \in R$, then there exists an element $z$ such that $z \in X$ and $\langle x, z\rangle,\langle z, y\rangle \in R$. 
We say that $R$ is mediate if and only if

(Def. 6) $R$ is mediate in field $R$.

Let $R$ be a relational structure. We say that $R$ is mediate if and only if

(Def. 7) the internal relation of $R$ is mediate in the carrier of $R$.

Let us note that every relational structure which is reflexive is also mediate.

\section{Approximations Revisited}

Now we state the proposition:

(5) Let us consider a non empty relational structure $R$ and elements $a, b$ of $R$. Suppose $a \in \operatorname{UAp}(\{b\})$. Then $\langle a, b\rangle \in$ the internal relation of $R$.

Let $R$ be a non empty relational structure and $X$ be a subset of $R$. The functor $\operatorname{Uap} X$ yielding a subset of $R$ is defined by the term

(Def. 8) $\left(\operatorname{LAp}\left(X^{\mathrm{c}}\right)\right)^{\mathrm{c}}$.

The functor Lap $X$ yielding a subset of $R$ is defined by the term

(Def. 9) $\left(\operatorname{UAp}\left(X^{\mathrm{c}}\right)\right)^{\mathrm{c}}$

Now we state the propositions:

(6) Let us consider a non empty relational structure $R$, a subset $X$ of $R$, and an element $x$. If $x \in \operatorname{LAp}(X)$, then $[x]_{\alpha} \subseteq X$, where $\alpha$ is the internal relation of $R$.

(7) Let us consider a non empty relational structure $R$, a subset $X$ of $R$, and a set $x$. If $x \in \operatorname{UAp}(X)$, then $[x]_{\alpha}$ meets $X$, where $\alpha$ is the internal relation of $R$.

Let us consider a non empty relational structure $R$ and a subset $X$ of $R$. Now we state the propositions:

(8) $\operatorname{Uap} X=\operatorname{UAp}(X)$.

(9) $\operatorname{Lap} X=\operatorname{LAp}(X)$.

Let us consider a non empty void relational structure $R$ and a subset $X$ of $R$. Now we state the propositions:

(10) $\operatorname{LAp}(X)=\Omega_{R}$.

(11) $\operatorname{UAp}(X)=\emptyset_{R}$.

\section{General Properties of Approximations}

Let $R$ be a non empty relational structure. Observe that $\operatorname{LAp}\left(\Omega_{R}\right)$ reduces to $\Omega_{R}$.

Let $R$ be a non empty serial relational structure. One can check that $\operatorname{UAp}\left(\Omega_{R}\right)$ reduces to $\Omega_{R}$. 
One can check that $\operatorname{LAp}\left(\emptyset_{R}\right)$ reduces to $\emptyset_{R}$.

Let $R$ be a non empty relational structure. Note that $\operatorname{UAp}\left(\emptyset_{R}\right)$ reduces to $\emptyset_{R}$.

Let us consider a non empty relational structure $R$ and subsets $X, Y$ of $R$. Now we state the propositions:

(12) $\operatorname{LAp}(X \cap Y)=\operatorname{LAp}(X) \cap \operatorname{LAp}(Y)$.

(13) $\operatorname{UAp}(X \cup Y)=\operatorname{UAp}(X) \cup \operatorname{UAp}(Y)$.

(14) If $X \subseteq Y$, then $\operatorname{LAp}(X) \subseteq \operatorname{LAp}(Y)$.

(15) If $X \subseteq Y$, then $\operatorname{UAp}(X) \subseteq \operatorname{UAp}(Y)$.

Now we state the propositions:

(16) Let us consider a non empty relational structure $R$ and a subset $X$ of $R$. Then $\operatorname{LAp}\left(X^{\mathrm{c}}\right)=(\operatorname{UAp}(X))^{\mathrm{c}}$.

(17) Let us consider a non empty serial relational structure $R$ and a subset $X$ of $R$. Then $\operatorname{LAp}(X) \subseteq \operatorname{UAp}(X)$.

\section{Auxiliary Operations on Approximation Operators}

Let $R$ be a non empty relational structure. The functors $\operatorname{LAp}(R)$ and $\operatorname{UAp}(R)$ yielding functions from $2^{\text {the carrier of } R}$ into $2^{\text {the carrier of } R}$ are defined by the conditions, respectively.

(Def. 10) Let us consider a subset $X$ of $R$. Then $(\operatorname{LAp}(R))(X)=\operatorname{LAp}(X)$.

(Def. 11) Let us consider a subset $X$ of $R$. Then $(\operatorname{UAp}(R))(X)=\operatorname{UAp}(X)$.

Let $A$ be a non empty set and $U$ be a function from $2^{A}$ into $2^{A}$. We say that $U$ preserves empty set if and only if

(Def. 12) $U(\emptyset)=\emptyset$.

We say that $U$ preserves universe if and only if

(Def. 13) $U(A)=A$.

Observe that $\mathrm{id}_{2 A}$ preserves empty set and universe as a function from $2^{A}$ into $2^{A}$.

One can verify that there exists a function from $2^{A}$ into $2^{A}$ which preserves empty set and universe.

Let $X$ be a set and $f$ be a function from $2^{X}$ into $2^{X}$. The functor Flip $f$ yielding a function from $2^{X}$ into $2^{X}$ is defined by

(Def. 14) Let us consider a subset $x$ of $X$. Then $i t(x)=f\left(x^{\mathrm{c}}\right)^{\mathrm{c}}$.

Let us consider a set $X$ and a function $f$ from $2^{X}$ into $2^{X}$. Now we state the propositions:

(18) If $f(\emptyset)=\emptyset$, then $($ Flip $f)(X)=X$.

(19) If $f(X)=X$, then $($ Flip $f)(\emptyset)=\emptyset$.

(20) If $f=\operatorname{id}_{2 X}$, then Flip $f=f$. 
Let us consider a set $X$, a function $f$ from $2^{X}$ into $2^{X}$, and subsets $A, B$ of $X$. Now we state the propositions:

(21) If for every subsets $A, B$ of $X, f(A \cup B)=f(A) \cup f(B)$, then (Flip $f)(A \cap$ $B)=(\operatorname{Flip} f)(A) \cap($ Flip $f)(B)$.

(22) If for every subsets $A, B$ of $X, f(A \cap B)=f(A) \cap f(B)$, then (Flip $f)(A \cup$ $B)=(\operatorname{Flip} f)(A) \cup(\operatorname{Flip} f)(B)$.

Now we state the proposition:

(23) Let us consider a set $X$ and a function $f$ from $2^{X}$ into $2^{X}$. Then Flip Flip $f$ $=f$. Proof: Set $g=$ Flip Flip $f$. For every subset $x$ of $X, g(x)=f(x)$.

Let $A$ be a non empty set and $f$ be a function from $2^{A}$ into $2^{A}$. Observe that Flip $f$ preserves empty set.

Let $f$ be a function from $2^{A}$ into $2^{A}$. One can verify that Flip $f$ preserves universe.

Now we state the proposition:

(24) Let us consider a non empty set $A$ and functions $L, U$ from $2^{A}$ into $2^{A}$. Suppose

(i) $U=$ Flip $L$, and

(ii) for every subset $X$ of $A, L(L(X)) \subseteq L(X)$.

Let us consider a subset $X$ of $A$. Then $U(X) \subseteq U(U(X))$.

\section{Towards Topological Models of Rough Sets}

Let $T$ be a topological space. The functors ClMap $T$ and $\operatorname{IntMap} T$ yielding functions from $2^{\text {the carrier of } T}$ into $2^{\text {the carrier of } T}$ are defined by the conditions, respectively.

(Def. 15) Let us consider a subset $X$ of $T$. Then $(\operatorname{ClMap} T)(X)=\bar{X}$.

(Def. 16) Let us consider a subset $X$ of $T$. Then $(\operatorname{IntMap} T)(X)=\operatorname{Int} X$.

Let $f$ be a function from $2^{\text {the carrier of } T}$ into $2^{\text {the carrier of } T}$. We say that $f$ is closed-valued if and only if

(Def. 17) Let us consider a subset $X$ of $T$. Then $f(X)$ is closed.

We say that $f$ is open-valued if and only if

(Def. 18) Let us consider a subset $X$ of $T$. Then $f(X)$ is open.

Note that ClMap $T$ is closed-valued and $\operatorname{IntMap} T$ is open-valued.

Let us observe that there exists a function

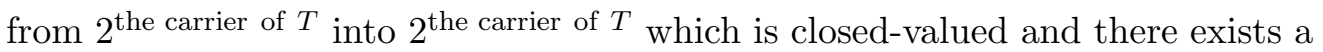
function from $2^{\text {the carrier of } T}$ into $2^{\text {the carrier of } T}$ which is open-valued.

Let us consider a topological space $T$. Now we state the propositions:

Flip ClMap $T=\operatorname{IntMap} T$. 
(26) Flip IntMap $T=\operatorname{ClMap} T$.

Let $T$ be a non empty topological space. One can verify that ClMap $T$ preserves empty set and universe and IntMap $T$ preserves empty set and universe.

\section{Formalization of Zhu's Paper [19]}

Let us consider a non empty relational structure $R$. Now we state the propositions:

(27) $\quad$ Flip $\operatorname{UAp}(R)=\operatorname{LAp}(R)$.

(28) $\operatorname{Flip} \operatorname{LAp}(R)=\operatorname{UAp}(R)$.

Now we state the proposition:

(29) Let us consider a non empty finite set $A$ and a function $U$ from $2^{A}$ into $2^{A}$. Suppose

(i) $U(\emptyset)=\emptyset$, and

(ii) for every subsets $X, Y$ of $A, U(X \cup Y)=U(X) \cup U(Y)$.

Then there exists a non empty finite relational structure $R$ such that

(iii) the carrier of $R=A$, and

(iv) $U=\operatorname{UAp}(R)$.

The theorem is a consequence of (13). Proof: Define $\mathcal{P}$ [set, set $] \equiv \$_{1} \in$ $L\left(\left\{\$_{2}\right\}\right)$. Consider $R$ being a binary relation on $A$ such that for every elements $x, y$ of $A,\langle x, y\rangle \in R$ iff $\mathcal{P}[x, y]$. Reconsider $R R=\langle A, R\rangle$ as a non empty finite relational structure. For every element $y$ of $R R$ and for every subset $Y$ of $R R$ such that $Y=\{y\}$ holds $\operatorname{UAp}(Y)=L(Y)$. For every element $x$ such that $x \in \operatorname{dom} \operatorname{UAp}(R R)$ holds $(\operatorname{UAp}(R R))(x)=L(x)$.

Let us consider a non empty finite set $A$ and a function $L$ from $2^{A}$ into $2^{A}$. Now we state the propositions:

(30) Suppose $L(A)=A$ and for every subsets $X, Y$ of $A, L(X \cap Y)=$ $L(X) \cap L(Y)$. Then there exists a non empty finite relational structure $R$ such that

(i) the carrier of $R=A$, and

(ii) $L=\operatorname{LAp}(R)$.

(31) Suppose $L(A)=A$ and $L(\emptyset)=\emptyset$ and for every subsets $X, Y$ of $A$, $L(X \cap Y)=L(X) \cap L(Y)$. Then there exists a non empty serial relational structure $R$ such that

(i) the carrier of $R=A$, and

(ii) $L=\operatorname{LAp}(R)$.

Now we state the propositions: 
(32) Let us consider a non empty finite set $A$ and a function $U$ from $2^{A}$ into $2^{A}$. Suppose

(i) $U(A)=A$, and

(ii) $U(\emptyset)=\emptyset$, and

(iii) for every subsets $X, Y$ of $A, U(X \cup Y)=U(X) \cup U(Y)$.

Then there exists a non empty finite serial relational structure $R$ such that

(iv) the carrier of $R=A$, and

(v) $U=\operatorname{UAp}(R)$.

The theorem is a consequence of (29). Proof: Consider $R$ being a non empty finite relational structure such that the carrier of $R=A$ and $U=$ $\operatorname{UAp}(R)$. For every element $x$ such that $x \in$ the carrier of $R$ there exists an element $y$ such that $y \in$ the carrier of $R$ and $\langle x, y\rangle \in$ the internal relation of $R$.

(33) Let us consider a non empty finite set $A$ and a function $L$ from $2^{A}$ into $2^{A}$. Suppose

(i) $L(A)=A$, and

(ii) for every subset $X$ of $A, L(X) \subseteq L\left(X^{\mathrm{c}}\right)^{\mathrm{c}}$, and

(iii) for every subsets $X, Y$ of $A, L(X \cap Y)=L(X) \cap L(Y)$.

Then there exists a non empty finite serial relational structure $R$ such that

(iv) the carrier of $R=A$, and

(v) $L=\operatorname{LAp}(R)$.

The theorem is a consequence of (30). Proof: Consider $R$ being a non empty finite relational structure such that the carrier of $R=A$ and $L=$ $\operatorname{LAp}(R)$. For every element $x$ such that $x \in$ the carrier of $R$ there exists an element $y$ such that $y \in$ the carrier of $R$ and $\langle x, y\rangle \in$ the internal relation of $R$.

(34) Let us consider a non empty finite set $A$ and a function $U$ from $2^{A}$ into $2^{A}$. Suppose

(i) $U(\emptyset)=\emptyset$, and

(ii) for every subset $X$ of $A, U\left(X^{\mathrm{c}}\right)^{\mathrm{c}} \subseteq U(X)$, and

(iii) for every subsets $X, Y$ of $A, U(X \cup Y)=U(X) \cup U(Y)$.

Then there exists a non empty serial relational structure $R$ such that

(iv) the carrier of $R=A$, and

(v) $U=\operatorname{UAp}(R)$. 
The theorem is a consequence of (29), (19), and (27). Proof: Consider $R$ being a non empty finite relational structure such that the carrier of $R=A$ and $U=\operatorname{UAp}(R)$. For every element $x$ such that $x \in$ the carrier of $R$ there exists an element $y$ such that $y \in$ the carrier of $R$ and $\langle x$, $y\rangle \in$ the internal relation of $R$.

Let us consider a non empty reflexive relational structure $R$ and a subset $X$ of $R$. Now we state the propositions:

(36) $X \subseteq \operatorname{UAp}(X)$.

Now we state the propositions:

(37) Let us consider a non empty finite set $A$ and a function $U$ from $2^{A}$ into $2^{A}$. Suppose

(i) $U(\emptyset)=\emptyset$, and

(ii) for every subset $X$ of $A, X \subseteq U(X)$, and

(iii) for every subsets $X, Y$ of $A, U(X \cup Y)=U(X) \cup U(Y)$.

Then there exists a non empty finite reflexive relational structure $R$ such that

(iv) the carrier of $R=A$, and

(v) $U=\operatorname{UAp}(R)$.

The theorem is a consequence of (32). Proof: Consider $R$ being a non empty finite serial relational structure such that the carrier of $R=A$ and $U=\operatorname{UAp}(R)$. For every element $x$ such that $x \in$ the carrier of $R$ holds $\langle x, x\rangle \in$ the internal relation of $R$.

(38) Let us consider a non empty finite set $A$ and a function $L$ from $2^{A}$ into $2^{A}$. Suppose

(i) $L(A)=A$, and

(ii) for every subset $X$ of $A, L(X) \subseteq X$, and

(iii) for every subsets $X, Y$ of $A, L(X \cap Y)=L(X) \cap L(Y)$.

Then there exists a non empty finite reflexive relational structure $R$ such that

(iv) the carrier of $R=A$, and

(v) $L=\operatorname{LAp}(R)$.

The theorem is a consequence of (19), (22), (37), (23), and (27). Proof: Set $U=$ Flip $L$. For every subset $X$ of $A, X \subseteq U(X)$. Consider $R$ being a non empty finite reflexive relational structure such that the carrier of $R=A$ and $U=\operatorname{UAp}(R)$. 
Let us consider a non empty mediate relational structure $R$ and a subset $X$ of $R$. Now we state the propositions:

(39) $\operatorname{UAp}(X) \subseteq \operatorname{UAp}(\operatorname{UAp}(X))$.

(40) $\operatorname{LAp}(\operatorname{LAp}(X)) \subseteq \operatorname{LAp}(X)$.

Now we state the proposition:

(41) Let us consider a non empty finite set $A$ and a function $U$ from $2^{A}$ into $2^{A}$. Suppose

(i) $U(\emptyset)=\emptyset$, and

(ii) for every subset $X$ of $A, U(X) \subseteq U(U(X))$, and

(iii) for every subsets $X, Y$ of $A, U(X \cup Y)=U(X) \cup U(Y)$.

Then there exists a non empty mediate finite relational structure $R$ such that

(iv) the carrier of $R=A$, and

(v) $U=\operatorname{UAp}(R)$.

The theorem is a consequence of (29) and (5). Proof: Consider $R$ being a non empty finite relational structure such that the carrier of $R=A$ and $U=\operatorname{UAp}(R)$. For every elements $x, y$ such that $x, y \in$ the carrier of $R$ holds if $\langle x, y\rangle \in$ the internal relation of $R$, then there exists an element $z$ such that $z \in$ the carrier of $R$ and $\langle x, z\rangle,\langle z, y\rangle \in$ the internal relation of $R$.

Let us consider a non empty finite set $A$ and a function $L$ from $2^{A}$ into $2^{A}$. Now we state the propositions:

(42) Suppose $L(A)=A$ and for every subset $X$ of $A, L(L(X)) \subseteq L(X)$ and for every subsets $X, Y$ of $A, L(X \cap Y)=L(X) \cap L(Y)$. Then there exists a non empty mediate finite relational structure $R$ such that

(i) the carrier of $R=A$, and

(ii) $L=\operatorname{LAp}(R)$.

(43) Suppose $L(A)=A$ and for every subsets $X, Y$ of $A, L(X \cap Y)=$ $L(X) \cap L(Y)$. Then for every subset $X$ of $A, L(X) \subseteq L\left(X^{\mathrm{c}}\right)^{\mathrm{c}}$ if and only if $L(\emptyset)=\emptyset$.

Now we state the proposition:

(44) Let us consider a non empty finite set $A$ and a function $U$ from $2^{A}$ into $2^{A}$. Suppose

(i) $U(\emptyset)=\emptyset$, and

(ii) for every subsets $X, Y$ of $A, U(X \cup Y)=U(X) \cup U(Y)$.

Then for every subset $X$ of $A, U\left(X^{\mathrm{c}}\right)^{\mathrm{c}} \subseteq U(X)$ if and only if $U(A)=A$. The theorem is a consequence of (34), (32), (27), and (17). 


\section{REFERENCES}

[1] Czesław Byliński. Functions and their basic properties. Formalized Mathematics, 1(1): 55-65, 1990.

[2] Czesław Byliński. Functions from a set to a set. Formalized Mathematics, 1(1):153-164, 1990.

[3] Czesław Byliński. Partial functions. Formalized Mathematics, 1(2):357-367, 1990.

[4] Czesław Byliński. Some basic properties of sets. Formalized Mathematics, 1(1):47-53, 1990.

[5] Agata Darmochwał. Finite sets. Formalized Mathematics, 1(1):165-167, 1990.

[6] Adam Grabowski. Basic properties of rough sets and rough membership function. Formalized Mathematics, 12(1):21-28, 2004.

[7] Adam Grabowski and Magdalena Jastrzębska. A note on a formal approach to rough operators. In Marcin S. Szczuka and Marzena Kryszkiewicz et al., editors, Rough Sets and Current Trends in Computing - 7th International Conference, RSCTC 2010, Warsaw, Poland, June 28-30, 2010. Proceedings, volume 6086 of Lecture Notes in Computer Science, pages 307-316. Springer, 2010. doi:10.1007/978-3-642-13529-3_33

[8] Artur Korniłowicz. Cartesian products of relations and relational structures. Formalized Mathematics, 6(1):145-152, 1997.

[9] Beata Padlewska and Agata Darmochwał. Topological spaces and continuous functions. Formalized Mathematics, 1(1):223-230, 1990.

[10] Z. Pawlak. Rough sets. International Journal of Parallel Programming, 11:341-356, 1982. doi:10.1007/BF01001956

[11] Konrad Raczkowski and Paweł Sadowski. Equivalence relations and classes of abstraction. Formalized Mathematics, 1(3):441-444, 1990.

[12] Andrzej Skowron and Jarosław Stepaniuk. Tolerance approximation spaces. Fundamenta Informaticae, 27(2/3):245-253, 1996. doi:10.3233/FI-1996-272311

[13] Andrzej Trybulec. Domains and their Cartesian products. Formalized Mathematics, 1(1): $115-122,1990$.

[14] Wojciech A. Trybulec and Grzegorz Bancerek. Kuratowski - Zorn lemma. Formalized Mathematics, 1(2):387-393, 1990.

[15] Zinaida Trybulec. Properties of subsets. Formalized Mathematics, 1(1):67-71, 1990.

[16] Edmund Woronowicz. Relations and their basic properties. Formalized Mathematics, 1 (1):73-83, 1990.

[17] Mirosław Wysocki and Agata Darmochwał. Subsets of topological spaces. Formalized Mathematics, 1(1):231-237, 1990.

[18] Y.Y. Yao. Two views of the theory of rough sets in finite universes. International Journal of Approximate Reasoning, 15(4):291-317, 1996. doi:10.1016/S0888-613X(96)00071-0

[19] William Zhu. Generalized rough sets based on relations. Information Sciences, 177: 4997-5011, 2007. 